\title{
Minimizing Noise in Pediatric Task-Based functional MRI; Adolescents with Developmental Disabilities and Typical Development
}

\author{
Catherine Fassbender ${ }^{1,2,3}$, Prerona Muhkerjee ${ }^{1,2}$, and Julie B. Schweitzer ${ }^{1,2}$ \\ ${ }^{1}$ Department of Psychiatry and Behavioral Sciences \\ ${ }^{2}$ UC Davis MIND Institute \\ ${ }^{3}$ UC Davis Imaging Research Center
}

\begin{abstract}
Functional Magnetic Resonance Imaging (fMRI) represents a powerful tool with which to examine brain functioning and development in typically developing pediatric groups as well as children and adolescents with clinical disorders. However, fMRI data can be highly susceptible to misinterpretation due to the effects of excessive levels of noise, often related to head motion. Imaging children, especially with developmental disorders, requires extra considerations related to hyperactivity, anxiety and the ability to perform and maintain attention to the fMRI paradigm. We discuss a number of methods that can be employed to minimize noise, in particular movementrelated noise. To this end we focus on strategies prior to, during and following the data acquisition phase employed primarily within our own laboratory. We discuss the impact of factors such as experimental design, screening of potential participants and pre-scan training on head motion in our adolescents with developmental disorders and typical development. We make some suggestions that may minimize noise during data acquisition itself and finally we briefly discuss some current processing techniques that may help to identify and remove noise in the data. Many advances have been made in the field of pediatric imaging, particularly with regard to research involving children with developmental disorders. Mindfulness of issues such as those discussed here will ensure continued progress and greater consistency across studies.
\end{abstract}

\section{Keywords}

fMRI; children; adolescents; developmental disability; head motion; noise

fMRI data are susceptible to noise from multiple sources (Greve et al., 2013). These include inhomogeneities in the magnetic field and physiological processes such as cardiac and respiratory cycles (Sarkka et al., 2012). Nevertheless, subject motion presents the largest

Catherine Fassbender, Ph.D., cfassbender@ucdavis.edu, Ph : (1) 916-703-0265, UC Davis MIND Institute, 2825 50th St., UC Davis Medical Center, Sacramento, CA 95817.

Publisher's Disclaimer: This is a PDF file of an unedited manuscript that has been accepted for publication. As a service to our customers we are providing this early version of the manuscript. The manuscript will undergo copyediting, typesetting, and review of the resulting proof before it is published in its final citable form. Please note that during the production process errors may be discovered which could affect the content, and all legal disclaimers that apply to the journal pertain. 
source of noise. Motion artifacts represent one of the largest confounding problems in MRI research. Friston et al (Friston et al., 1996) estimate that 30 to $90 \%$ of MRI noise can be attributed to movement. Noise from motion can confound the task signal by appearing as change in MRI signal intensity, as shift in relative position of an activated voxel can appear as if the value of the voxel changed. This is particularly problematic if the movement is correlated with the task parameters themselves (Epstein et al., 2007), as in that case, regressing out the motion will also regress out any brain signal changes associated with the task itself (Weinberger and Radulescu, 2016). Certain groups, such as pediatric and clinical populations, are particularly vulnerable to movement during scanning (Poldrack et al., 2002). Our research frequently centers on children with Attention Deficit Hyperactivity Disorder (ADHD), a particularly challenging group in terms of within-scanner head motion (see Table 1). ADHD Combined presentation is defined by developmentally inappropriate levels of hyperactivity. As researchers are frequently interested in observing the brain in its "natural" state, children are often asked to abstain from their ADHD medication until the plasma levels of the drug are no longer detectable. Thus, adding to the already elevated tendency of children to be active, compared to their adult counterparts, developmentally inappropriate levels of hyperactivity can represent a very challenging group in terms of MRI data quality.

There are a number of techniques that can be implemented to minimize artifactual findings or noisy data in pediatric groups. As movement represents one of the greatest contributors to noise in fMRI data, particularly in pediatric groups, the larger focus of this paper will be movement related noise. Focusing primarily on strategies that we utilize in our laboratory, we will discuss 1) factors to consider during the experimental design phase; 2) strategies to minimize movement during the scan through the use of hardware, training and relaxation techniques; and 3) data processing methodology. Other papers have focused on factors such as the developmental differences in anatomy and physiology (Kotsoni et al., 2006) and hemodynamic response function in pediatric groups, particularly infants (Poldrack et al., 2002) but this is beyond the scope of the current paper. Furthermore, we will restrict our discussion to task/GLM-related fMRI, rather than resting state or connectivity techniques.

\section{Experimental Design}

There are many factors to consider when designing a paradigm for pediatric groups (Davidson et al., 2003; Luna et al., 2010). Usually these are factors that are also prudent to consider when designing experiments for clinical and adult groups, but can have a pronounced impact on the levels of movement in children. One of the simplest design considerations that could make a significant impact on movement is the length of the MRI paradigm. Children tend to lose focus faster than most adults (Van Horn and Pelphrey, 2015), become more restless with time and move more in the scanner as a result. A study examining simultaneous EEG/fMRI recording in children between the ages of 6 and 18 (Centeno et al., 2016) examined movement across 4 10-minute blocks. The authors found a $100 \%$ increase in movement in the fourth compared to the first block. We examined movement in one of our longest MRI paradigms between the first and last block (each block is 6 minutes and $4 \mathrm{~s}$ in length). We also found significant increases in motion in all planes (See Table 2) and also in the first derivative of head displacement in block 4 compared with 
block 1 (calculated using AFNI's enorm function which calculates how much the head moved in one TR with respect to previous TR; ADHD: $\mathrm{t}(71)=3.76, \mathrm{p}=0.01$; $\mathrm{TD}$ : $(\mathrm{t}(84)=$ $2.11, \mathrm{p}=0.02)$. The sustained attention and vigilance literature has demonstrated evidence for a decline in attention and performance over time; for example, Helton et al (Helton et al., 2005 ) found a significant decline in attention occurring following about 30 minutes of performing a simulated air traffic control paradigm and Grier (Grier et al., 2003) et al found a steady decline in performance over time (measured in 10 minute intervals up to 50 minutes of task time) in their vigilance task. Recent evidence from our laboratory suggests that children with ADHD may increase their levels of activity as a compensatory mechanism to modulate attention and arousal (Hartanto et al., 2016). Therefore longer paradigms may be even more problematic for children with developmental disorders, although this was not supported by significant motion differences in our analysis reported above. Thus an optimal pediatric fMRI paradigm will balance considerations relating to numbers of analyzable events with maintaining an overall short imaging session to minimize motion, especially towards the end of the task.

Another consideration is the length of each individual task run; our laboratory and others suggest individual runs of about 4 to 5 minutes depending on the task (Davidson et al., 2003). Frequent breaks are useful to incorporate in the overall paradigm design. It is also important to keep the overall length of time spent in the scan environment to a minimum. The optimal length of time for an overall imaging session depends upon the degree that the paradigm/s engages participants. In our laboratory we ideally keep sessions to about 60 minutes in length, but with the inclusion of engaging paradigms we have successfully acquired reasonable data from pediatric and clinical groups with a session length of 90 minutes. It is tempting to attempt to maximize data collection from an individual once they are present and participating in a research study; however, the inclusion of extra structural, resting state DTI and additional functional paradigms will also increase boredom, frustration and the likelihood of increased movement. If additional scans are required it is advisable to conduct two separate imaging sessions on separate days or at least with a long break between (e.g., a lunch break). This keeps the sessions more interesting for the children and increases the likelihood that they will remain still, perform well, and participate in future studies. Another strategy is to play preferred music or movie clips during structural scans (e.g. MPRAGE, DTI) to give participants a mental break and keep them engaged (Poldrack et al., 2002).

A second consideration is the age-appropriateness of a task; paradigms that are too difficult or not sufficiently engaging might also increase movement due to boredom or lack of engagement in children (Davidson et al., 2003; Galvan, 2010; Galvan et al., 2012). Some researchers suggest using a "visual road map" in order to inform participants about their progression through the MRI study (Schlund et al., 2011). Furthermore, children should not be treated as a monolithic group; a child of 5 has very different cognitive capabilities and preferences compared with a 9-year-old child and a 9-year old differs from a 13-year old (Luna et al., 2010). Therefore, even a four-year age gap can represent a significant developmental span in children. An alternate approach to examining discrete age-groups is to add age as a continuous variable and examine children within wider age ranges (Galvan et al., 2012). If incentives are being used within the paradigm, either to encourage optimal 
performance, or as the object of study (measuring response to incentives themselves), the incentives utilized should be developmentally appropriate (Luking et al., 2016; Schweitzer and Sulzer-Azaroff, 1988, 1995). Careful consideration needs to be paid to whether or not the child understands the paradigm, is engaged consistent with the construct of interest for optimal, interpretable functional brain activity. In addition, the child's attention span, level of engagement and movement also change with development (Davidson et al., 2003; Poldrack et al., 2002). As mentioned in the introduction, movement that is correlated with the variable of interest (for example head nodding associated with pushing a button in response to the stimulus set of interest or head motion following a performance error (Epstein et al., 2007)) can pose particular problems; if the motion is correlated with a variable of interest regressing out movement parameters, a common step in MRI analysis, will remove activity associated with the variable of interest along with the movement. Thus it is critical to examine the data for such correlations to determine the optimal analysis strategy - for instance, whether or not these subjects should be eliminated (Bright and Murphy, 2015). These factors may become even more important with pediatric clinical groups such as children with ADHD or ASD, who tend to have greater issues with hyperactivity and generally, have a lower tolerance threshold for frustration than their typically developing peers. Therefore, it is always prudent to carefully examine the data for any factors that may be group-specific, as this may lead to false-positive, between-groups differences that are related to movement issues, artifact and noise, rather than real datadriven differences.

\section{Pre-scan screening to prevent and reduce movement concerns}

Both children and their parents may feel anxious or uneasy about the scan, as it represents an extremely unfamiliar situation. A factor that cannot be discounted is the parent's attitude towards the scan; children often sense a parent's reluctance and nervousness about MRI and this can trigger their anxiety. Therefore, an important first step during pediatric recruitment is to make the parent feel at ease. The parent should be encouraged to voice any concerns or ask any clarifying questions that they need to inform themselves about the safety of their child. However, as Galvan and colleagues (Galvan et al., 2012) point out, it is ill advised to rely on the parent's input too much at the expense of interacting with the child, in particular older children and adolescents; it is possible that the parent may not be aware of all of the adolescent participant's behaviors, attitudes, and feelings or that the adolescent may have, piercings and other medical issues that might preclude them from being scanned (for instance pregnancy, or an IUD in female participants). We find that children often have less fear associated with, and fewer preconceived notions about the scanner than adults, including a lower likelihood of negative association between scanners and urgent medical problems. Children should be screened for anxiety and phobia, including a fear of enclosed spaces during the initial evaluation phase for inclusion in an imaging study. Young children, for example, younger than 9 years of age, may not be able to articulate their fears, however. We recommend asking a few general questions regarding a prior history of fear of enclosed spaces with the parent and older children. Children should be reminded that they would be able to stop the scan at any time. Children may actually experience less emotional distress 
and greater enjoyment and satisfaction with participating in functional MRI studies based on a survey of 50 children and adolescents and 43 adults (Thomason, 2009).

\section{Strategies to minimize movement during the scan}

\section{Pre-scan training}

The next step is to familiarize the child with the fMRI method. A presentation with pictures of the MRI scanner and laying out the procedure for the child is very useful and employed by many laboratories. The scanner is sometimes "decorated" with child-friendly decorations to make it appear as a spaceship or other non-medical looking device (Nordahl et al., 2016). Other programs may use prerecorded videos of other children being screened for metal, entering a scanning environment, being placed in the scanner and performing a task to be used in the scanner (Thomason, 2009). The research participant can then be asked to briefly review what they observed in the pictures or video (Nordahl et al., 2016) and asked if they have any questions about the situation before proceeding. In our laboratory we also inform the participants that they will receive a t-shirt with a printed image of their own brain. The tshirt appears to be highly motivating and may assuage some of the fears experienced by the pediatric participants. Following the presentation it is useful to train pediatric participants in a mock scan environment and/or show them the actual scanner itself. This is preferably carried out on a day prior to the scan itself, at the time of assent/consent or completion of assessments and/or behavioral paradigms. This allows the researchers to determine, ahead of time, how comfortable the child will be in the scanner and how much they might be expected to move. In our laboratory we use the threshold of half a voxel size to train our participants on limiting movement within the mock scanner. The mock scanner environment also allows one to determine how much support the child will need and affords an extra opportunity to build a rapport (Galvan et al., 2012), which is essential for putting the child at ease and ultimately reducing movement.

Implementing training to teach children levels of acceptable and unacceptable movement will also improve the quality of the data acquired. Many young children will not understand the degree to which they are moving and may forget that they were asked to remain still as the task progresses. Training in mock scanners prior to the fMRI session has consistently shown to improve the quality of data acquisition in pediatric groups (de Amorim e Silva et al., 2006; De Bie, 2010; Kotsoni et al., 2006; Poldrack et al., 2002) and pediatric clinical groups (Epstein et al., 2007). Training often integrates methods from the applied behavior analytic (ABA) methodology (Nordahl et al., 2016; Slifer et al., 1993). Keith Slifer and colleagues pioneered the use of applied behavior analysis to reduce movement in the scanning environment with verbal feedback and a prize provided for improvements in head movement during mock scan training (Slifer et al., 1993; Slifer et al., 2002). The application of $\mathrm{ABA}$ procedures in the imaging context has become quite sophisticated including using such techniques as behavioral shaping, developing conditioned reinforcers associated with the scanning procedure, peer modeling, combined with systematic desensitization strategies. These methods allow researchers to obtain data of optimal quality from pediatric subjects (Nordahl et al., 2016). State-of-the-art ABA methods can be especially helpful when imaging children with developmental disorders such as autism spectrum disorders and 
intellectual impairment (Nordahl et al., 2016). Some laboratories furnish children with headsets that allow researchers to monitor their movement while they perform the practice tasks in the mock scanning environment. In our laboratory we provide visual feedback to our participants about their head movement while they are in the mock scanner; this allows them to experience how much head movement is excessive movement. Other laboratories have used a system whereby children are presented with a video game to train them to be still (http://www.currentstudios.ca/mri/; (Raschle et al., 2012) http://www.sacklerinstitute.org/ cornell/assaysandtools/). Children quickly learn that even small amounts of movement are unacceptable by employing this powerful reinforcer.

In the mock scanner, the child has an opportunity to experience the scan environment (Kotsoni et al., 2006). The sounds of the different imaging sequences (e.g. MPRAGE, EPI, DTI) can be played through a headphone system and they can practice the paradigm that they will subsequently perform in the actual scanner. This may be particularly important for pediatric clinical groups (Lucker et al., 1996). The mock scanner also provides a useful opportunity to train children on how to move if they need to; for instance if they need to scratch an itch. This can be practiced in the mock scanner showing them how to move slowly and carefully when unavoidable, or ideally in between scan runs. In hyperactive children it is also important to point out that movement of the limbs effects head movement; some children may not be aware that moving any part of their body can also cause their heads to move. Again, a good rapport with the child enables the researcher to explain why it is important to stay as still as possible to a receptive child and fosters a desire in the child to perform to their maximum potential. Children will also feel freer to express any concerns they might have. It is prudent to remain sensitive to and aware of the child's body language. If it appears that the participant is nervous or anxious it may be necessary to discuss this with the child. Relaxation techniques can be employed to reduce anxiety (Poldrack et al., 2002); however it is important to remain sensitive at all times to children whose anxiety levels exceed acceptable limits. It is unethical and unnecessary to subject a child to a situation in which they are extremely anxious to the point that they are experiencing distress. Finally, as mentioned previously, our laboratory and others have found it useful to play music (Slifer et al., 1991) or present a video, during the acquisition of the non-functional imaging scan itself, to further relax and engage participants during set up (placing the participant in the magnet) or "down time" in the scanner (Poldrack et al., 2002).

\section{Minimizing motion during image acquisition}

Once the participant has completed pre-scan training and is comfortable moving ahead with the scan, there are additional methods that can be employed to assist further in reducing head movement during data acquisition. Some laboratories use different hardware to forcibly keep the head still or to provide feedback as to when the child is moving. These methods range from inflatable pillows or foam cushions (Szaflarski et al., 2006) that envelop most of the head, to bite bars (Adjamian et al., 2004; Vaidya et al., 1998) and head restraints adapted from the medical field (Temple et al., 2001), to adhesive tape connecting the forehead and or the chin to the headcoil, as in prior work from our colleagues (Weissman et al., 2015). With regard to methods such as head restraints and bite bars, pediatric groups tend to have a low tolerance for this type of motion correction technique. Furthermore, any distress experienced 
as a result of such a technique may render the acquired functional MRI data questionable. We have developed a minimally invasive apparatus that is very well tolerated by our pediatric participants. Our system was inspired by Dr. Elliott Hong's bite bar system at the Maryland Psychiatric Research Center and University of Maryland School of Medicine (Hong et al., 2009) and employs a dental rest, on which participants place their upper incisors. A mold of each individual's dental impression (only the upper incisors) is attached to the mirror mount system used with the MRI headcoil. The mold does not protrude into the participant's mouth, and participants can speak, swallow and close their mouths around the dental rest with ease. This is critical for each participant's comfort. All of these factors increase compliance with the device and are very effective in reducing overall head motion. Few participants opt out of using the dental rest for reasons relating to comfort. In our longitudinal study, we encountered 127 out of 224 subjects who were scanned without the dental rest. Out of these, some participants were not offered the device due to technical reasons. Others were unable to use the device because their head size was large relative to the MRI headcoil casing. However, some individuals were not comfortable with, and thus refused to use the dental rest - this may be the most problematic group with respect to motion, as this might have been correlated with higher restlessness. To illustrate the effectiveness of the device in countering the impact of motion, we performed an analysis comparing movement parameters between two groups of individuals scanned with and without the dental rest. Due to technical reasons we conducted scans without offering the dental rest for a period of time. We randomly selected 48 individuals; 12 children with ADHD who had used the dental rest and 12 with ADHD who had not, 12 TD children who had used the dental rest and 12 who had not. We ran two sample t-tests for each of the six movement parameters (x, y, z, pitch, roll, yaw) to compare between individuals with and without dental rest within each diagnostic group. The movement parameters were extracted from the realignment step and absolute values of each parameter was taken for analysis. As we expected, when examining all participants together, the movement was higher in the group of individuals with no dental rest in dental rest group in $\mathrm{x}$ direction $(\mathrm{t}(46)=2.02 ; \mathrm{p}=$ $0.05)$ and a trend in that direction in the $\mathrm{z}$ plane $(\mathrm{p}=0.06)$. When we examined the groups separately, the TD group showed less movement in the dental rest group, along the $\mathrm{x}, \mathrm{y}, \mathrm{z}$, pitch, and roll, directions (see Table 3). Although the ADHD group without the dental rest did have elevated motions compared to the group with the dental rest, this did not reach significance (there was a trend in the pitch direction ( $\mathrm{p}=0.09$ ). See Table 3 for details. Differences in motion between the dental and no dental rest groups in the ADHD cohort did not reach significance perhaps due to elevated rates of variability in the ADHD no dental rest group.

Another important factor that can enhance data quality is real-time monitoring of the pediatric participant while in the scanner. This means verbally communicating with him/her at regular intervals, preferably between runs of the paradigm and checking that they are comfortable. Providing children with positive reinforcement, through the use of positive feedback about their performance, can also help to maintain their motivation to perform well. When possible, the researcher should monitor task performance and the relationship between task performance and head movement. Failure to respond to a number of successive stimuli and sudden head movements may be indicators of distress. Monitoring the 
participant's head movement should be performed either by visual examination of the EPI sequences in real time or by real-time movement feedback. It is also advisable to visually monitor their body movements from the operating room. Many laboratories, including ours, record large body movements in a scan log. The participant can be reminded between runs about minimizing body movements. With pediatric participants there is also an increased rate of requesting to exit the scanner, for instance to use the restroom, in the middle of a scan session. In this event it is important to go through shimming procedure before resuming the scan. An advantage of the dental rest system is that the head returns to a very similar position following such an event.

\section{Motion Correction during Data Processing}

Subject preparation and careful data acquisition should reduce the amount of movement. Nevertheless, there will be some remaining contamination due to motion, as a certain amount of movement during the scan is unavoidable. Movement that is randomly distributed across a task, is likely not to be biased towards one variable of interest and is likely to cancel out given sufficient number of subjects and scans. However, movement that is correlated with experimental factors (such as group or task), may confound the task response, and lead to false positive findings.

One of the most important and frequently neglected steps is to visually inspect all of the data at every stage of processing and correction. Using the video option in an image viewer (such as AFNI (https://afni.nimh.nih.gov (Cox, 1996)) or SPM (http://www.fil.ion.ucl.ac.uk/ spm(Vaidya et al., 1998))) all volumes should be sequentially scrolled through, looking for any sharp changes. Additionally, it is critical to examine the time series from voxels across the brain for outliers. Finally motion parameters and outliers should be compared between groups and conditions to look for significant differences. There are tools available for quality control and correction of fMRI data, such as ArtRepair (Mazaika et al., 2009; Mazaika et al., 2007), and @ss_review_basic and @ ss_review_driver generated by afni_proc.py in AFNI (Cox, 2012), for review see (Davids et al., 2014). Visual inspection is still important to spot qualitative anomalies. This is particularly important for pediatric and clinical populations, because there may be elevated degrees of movement and even more individual variation in brains and psychological processes. We focus inspection particularly at the boundaries of the brain, which are regions most susceptible to motion artifacts. Further we compare motion parameters and censor counts between the groups (and conditions) to ensure there are no significant differences. We will discuss quality control steps in some more detail below.

Extreme motion effects cannot be easily corrected, and data thus impacted should be eliminated from further analysis. Eliminating large amounts of intermediate data points might cause discontinuity in the data. This can be addressed by interpolating to infer the missing information from neighboring data points, and several tools are available for this purpose. However, the implications of such interpolation should be carefully considered. Furthermore, the output should be carefully examined. These considerations are especially true for populations with high movement (and potential irregularity), as these factors will cause a higher degree of guesswork, and consequently, greater scope for error. Therefore, in these cases, it is often advisable to eliminate that participant's data from further analysis. It 
is useful to decide a priori a reasonable cut-off point for accepting percentages of censored images per run and over the entire task in order to reduce bias. For instance in our analyses we have chosen a cut-off of $15 \%$; participants with more than $15 \%$ censored data are excluded from further analysis. We have chosen this cut-off based upon our calculation of the likelihood of having sufficient numbers of analyzable trials of interest within the remaining data (e.g., see (Murphy and Garavan, 2005; Turner and Miller, 2013) for suggestions on optimal trial numbers for fMRI research). This number may vary across laboratories depending upon the numbers of likely available trials of interest within their own experiment.

It is believed that moderate effects of motion can be programmatically corrected during preprocessing (Johnstone et al., 2006; Lund et al., 2005; Oakes et al., 2005) with different analysis packages having their own motion correction. Small degrees of motion may be more problematic for resting state fMRI and connectivity analyses but that discussion is beyond the scope of this paper. fMRI data is recorded in slices across the brain, and each slice is divided into voxels. Slices stack up to make one volume, with a series of volumes making up one run, and data being aggregated across volumes to make up response to stimulus. Thus, head movement between volumes presents a problem as the same voxel could move in the data space while recording, reflecting an inaccurate change in signal. This is addressed by aligning images by co-registration, and is performed in several stages. The first stage is realignment, which aligns all volumes from one subject. Following realignment the functional images from a participant are aligned to that individual's anatomical image. Finally all images from each subject are aligned to a standard coordinate space, so that they can be statistically compared (Friston et al., 1999). Usually all the transformations are calculated as parameters to be applied to each voxel (registration) and then applied cumulatively (reslicing). This process minimizes the errors that might result from each transformation. Other authors have discussed this in greater detail (e.g., (Beall and Lowe, 2014). These motion correction techniques assume that motion affects the entire volume in a similar fashion and attempt to address first-order motion effects; however, they do not address potential slice-to-slice out of plane motion effects or other second order motion effects. Such motion effects may arise from primarily spin history effects (out of plane motion (Friston et al., 1996; Muresan et al., 2005; Yancey et al., 2011), but also B1 inhomogeneity, and B0 inhomogeneity. Semi-prospective (e.g., SimPACE, (Beall and Lowe, 2014)) and retrospective (e.g., SLOMOCO, (Beall and Lowe, 2014) correction methods exist which attempt to address second order motion effects. Other methods of motion correction include independent components analysis (ICA; e.g., FMRIB's FIX see (Griffanti et al., 2014; Salimi-Khorshidi et al., 2014) and multi-echo fMRI (Bright and Murphy, 2013; Kundu et al., 2015). These methods have been shown to separate noise artifact from the BOLD signal. Prospective motion correction has also been utilized with some promise, for example MRI-compatible camera systems that measure head motion via a tracker mounted to the participant's head. Information about head motion in six planes is then fed back into the system in real time and is used to update the radio frequency pulses and gradients (Maclaren et al., 2013; Maziero et al., 2016; Todd et al., 2015).

Even after all these preprocessing steps, there is typically some residual effect of motion (as high as 30\% in a recent study (Beall and Lowe, 2014). These should be either addressed 
using advanced data processing tools or they can be controlled for in the statistical design by integrating motion metrics as nuisance regressors in the design matrix (Fox et al., 2009; Weissenbacher et al., 2009). These motion metrics may include motion parameters from the volume realignment step (Friston et al., 1996), first derivatives of these motion parameters or frame-wise displacement (FD). It may also include quadratic terms of the motion parameters and DVARS or the root mean squared change in BOLD signal from volume to volume (Power et al., 2013). It may also include spike information to effectively de-weight the TR's where excessive volume-to-volume movement occurred, which can be calculated with software packages (including ARTrepair for SPM, 3dDespike for AFNI, and fsl_motion_outliers for FSL). Further these nuisance regressors could be included in the design matrix either at the subject level, or at the group level to account for variance across subjects due to their motion (Beall and Lowe, 2014; Lund et al., 2005; Oakes et al., 2005). Some of these methods are discussed in detail in (Satterthwaite et al., 2013), especially in the context of resting state connectivity where they are even more important (Fox et al., 2009; Satterthwaite et al., 2013). However such motion parameter regression may also impact some of the task related signal when the motion is correlated with the experimental design, especially in block design tasks. Block designs are particularly susceptible to motion effects as head motion associated with potential reactions to stimuli or button presses to variables of interest can correlate highly with the sustained HRF within a block (Johnstone et al., 2006). If this correlation is low then the signal will be increased by this process and if the correlation is high then it will be decreased (Bright and Murphy, 2015; Bullmore et al., 1999; Churchill et al., 2012; Johnstone et al., 2006; Ollinger JM, 2009). Event-related designs can be less problematic (Birn et al., 1999); event-related paradigms tend to involve brief response to randomly temporally separated events of interest and the response-related motion will have a different temporal shape than the smooth HRF associated with the BOLD signal (Johnstone et al., 2006). Another problem with including these regressors in the design is that too many regressors at the first level reduces the degrees of freedom and may reduce the power (Bright and Murphy, 2015; Johnstone et al., 2006; Pernet, 2014; Siegel et al., 2014). An alternative might be to remove the data from subjects thus impacted.

Nevertheless, leaving out subjects for motion may cause bias in subject selection, especially for clinical groups such as ADHD or multiple sclerosis (Couvy-Duchesne et al., 2016; Wylie et al., 2014).

Although a detailed discussion of each preprocessing step is beyond the present scope, we will briefly describe some preprocessing steps employed in fMRI analyses. As these steps are not specific to pediatric groups we will only highlight factors specifically related to mitigating noise in pediatric groups.

Data are often examined for outlier data points and temporally smoothed. Outlier data points are defined by falling beyond a number of standard deviations from the mean for that voxel and this threshold can be adjusted based on the population of interest. For example, in our laboratory, we exclude participants with greater than $15 \%$ of the total number of volumes censored for our project. We have chosen this cut-off based upon our calculation of the likelihood of having sufficient numbers of analyzable trials of interest within the remaining data (Murphy and Garavan, 2005; Turner and Miller, 2013) for suggestions on optimal trial 
numbers for fMRI research). This number may vary across laboratories depending upon the numbers of likely available trials of interest within their own experiment.

Data are realigned by moving all volumes to overlay a chosen reference volume along six dimensions (i.e., translation along, the $\mathrm{x}, \mathrm{y}, \mathrm{z}$ axes or rotation around each axis. Volume-tovolume movements are referred to as first-order movement effects. These motion correction techniques assume that motion affects the entire volume in a similar fashion; however, however, they do not address potential slice-to-slice out of plane motion effects or other second order motion effects. Such motion effects may arise from primarily spin history effects (out of plane motion (Friston et al., 1996; Muresan et al., 2005; Yancey et al., 2011), but also B1 inhomogeneity, and B0 inhomogeneity. Very rapid head motion sometimes occurs within a TR. As Tierney et al (Tierney et al., 2016) illustrate, this can result in false positive activations. This may be particularly problematic in pediatric samples (Satterthwaite et al., 2013). As mentioned previously, there are a number of techniques that attempt to address second order motion effects (e.g., SLOMOCO, (Beall and Lowe, 2014); 2dlmReg and 3dDespike in AFNI; ArtRepair in SPM). Movement within a slice is less problematic as each slice is collected very rapidly (in about $100 \mathrm{msec}$ )(Wilke, 2003).

As the slices that make up a full volume of the brain are collected over an entire TR there will be a temporal lag between the acquisitions of different slices within a volume. Recent acquisition sequences such as GRAPPA can acquire two separate slices simultaneously, which lessens but does not eliminate the problem. Slice time correction attempts to correct for this.

As mentioned previously, the structural image for each participant is then warped onto a normalized template (e.g., Talairach or Montreal Neurological Institute. However, in some populations such as pediatric groups, individuals with disorders like schizophrenia, or healthy young or older adults, this could be problematic due to greater variability in anatomy as well as localization of functional activation (Brett et al., 2002; Park and Reuter-Lorenz, 2009). Thus approaches including population specific templates (Lee et al., 2005; Sanchez et al., 2012; Wilke et al., 2002, 2003; Zhang et al., 2014) study-specific templates, or subject matched templates (Rohlfing, 2012) have been suggested instead (Huang et al., 2010; Padilla et al., 2011). However, these might prevent generalization of results across studies, and the need for such approaches has also been questioned given that depending on the resolution and population this may not pose a big problem (Burgund et al., 2002; Kang et al., 2003) and many standard templates may contain enough variability to cause concern (For review, see (Evans et al., 2012; Mandal et al., 2012). Normalization to an adult template may be the most problematic when comparing children and adults, as the difference in head size may confound the group difference (Fonov et al., 2011).

Data sets are masked so that any spurious activity outside the brain is set to zero. However this should be carried out with caution and the data inspected for artifact (we will discuss this further in the section on Quality Control below). Activation might appear outside the brain due to multiple reasons including jaw clenching (muscle artifacts) or draining veins. We might also see noisy results, ghosting artifacts, and motion effects. Ghosting might result from scanner related issues. Large and highly significant clusters - may signal problems and 
should be more closely inspected. Certain artifacts (e.g., activity that resembles a straight line or big changes in signal intensity near the back or front of the brain) are not uncommon; they warrant additional quality control procedures and checking the data step by step to identify potential problem areas. An example of a bigger problem that might be disguised by masking is when a cluster originates outside the brain but close to the boundary of the brain. Such a cluster might have a small portion falling inside the brain boundary. Thus masking early will mask out the spurious activity outside the brain leaving only the activity inside the brain, making it look like a task related activation.

In addition to standard preprocessing steps there are other factors that should be considered with any MRI data set but particularly so with groups that are particularly susceptible to movement, such as pediatric groups.

\section{Quality Control}

The most important quality control step is to examine visually all the raw data and output from each preprocessing stage, due to the frequency of errors and prevalence of noise. Registration can be evaluated using a quality control script called ss_review_driver, which is created as a part of the afni_proc.py pipeline. In addition one must visually inspect the alignment. Alignment should be checked by comparing an individual subject's functional images with the anatomical volume, and then all subjects' anatomical images should be compared with each other. Subjects' anatomical images can be combined into one dataset and viewed as a video in the AFNI image viewer. In SPM, registration can be checked using the "Check reg" tool in the graphical interface. Further, in SPM, the origin of the image should be also checked by displaying the anatomical image using the display function and then making sure the 0,0,0 coordinates are at the anterior commissure. Although it can be difficult to quantify objective metrics as to what activity may be due to artifact, as the parameters vary based upon the population, task and brain regions of interest there are a number of patterns that can cause suspicion and may warrant further examination. 1) Large brain activations; 2) very intense activity outside the brain; 3 ) activity occurring on the borders of the brain or along the ventricles and brain regions susceptible to movement artifact may indicate problems with registration, may reflect excessive motion, external ghosting or scanner artifacts. Signs such as these should trigger a careful examination of the individual data set for problems with the registration (in which case the registration should be re-run with adjusted parameters) or a more careful examination of the motion parameters. If attempts to correct these errors do not succeed it may be necessary to exclude the subject as they could contaminate the group maps with non-task-related activations. Figure 1 illustrates a case of excessive motion and Figure 2 illustrates examples of bad registration taken from our dataset.

Apart from movement, other sources of noise can be from non-physiological causes, such as thermal noise due to distortion in current from heat induced electron movement, scanner drift or change in the field over time, inductance within the magnetic field by the brains own current distribution or non-uniformities in the scanner magnetic field (Huettel, Song \& McCarthy, 2009). Furthermore, artifacts can be from physiological signals such as cardiac and respiratory signals as head movements caused by heart beat or breathing, or changes in 
blood flow or blood oxygenation levels (Bhattacharyya and Lowe, 2004; Birn et al., 2004; Murphy et al., 2013).

We eliminate any data with extreme errors at every stage. Further, we control for residual errors by including the movement parameters (ie: the $\mathrm{x}, \mathrm{y}, \mathrm{z}$, pitch, roll, yaw measures from realignment stage) in our design matrix. It might also be suitable to use the first derivative of the motion parameters, or parameters derived from another tool that evaluates between volume movements or spikes. For a full discussion of framewise displacement (FD) measures see (Power et al., 2015) for a review. Briefly, differing methods of measuring FD are similar but differ in the amplitude of measurement and are influenced by the TR or sampling rate (more opportunities to record within-volume displacement, may result in better estimates of between-slice motion). Finally we compare the motion parameters, or other estimates of movement such as FD, between groups and conditions to test if movement differences are contributing to our results.

\section{Physiological Sources of Noise}

Apart from movement, other sources of noise include physiological signals such as cardiac and respiratory signals (Bhattacharyya and Lowe, 2004; Birn et al., 2006; Murphy, 2013). This can be especially problematic when it is correlated with the signal of interest, such as when the variable of interest increases breathing or heart rate, due to increased excitement or anxiety. Some of these signals are in a different frequency band and so get eliminated by band pass filtering, however as most fMRI data are acquired relatively slowly, some of this signal gets confounded with the neural signal (Bright and Murphy, 2015). This problem can be minimized by using multi-echo sequence, by including a short echo sequence simultaneously with the data (Bright and Murphy, 2013).

Several approaches can be taken to estimate physiological effects. Physiological data can be simultaneously collected with the fMRI data and included as nuisance regressors in the statistical model; however, in a similar vein to issues with task correlated motion, this might remove some pertinent brain signal (Murphy, 2013). Additionally, the collection of physiological data in children may be somewhat problematic as data are usually collected by means of monitors strapped to the chest and clipped to a finger, which may increase anxiety and discomfort in young subjects. Another method is to measure the signal from white matter or ventricles, as we expect that the neural activity should be mainly from the grey matter. These signals can then be used as proxies for physiological signals and regressed from the statistical model (Churchill et al., 2012). AFNI provides a tool 3dretroicor to estimate and process these signals and SPM also provides a tool called SPM DRIFTER (http://becs.aalto.fi/en/research/bayes/drifter/). Similarly, CompCor (http:// www.ncbi.nlm.nih.gov/pmc/articles/PMC2214855/) is used in the popular CONN functional connectivity toolbox to identify physiological signal in regions of non-interest (i.e. cerebrospinal fluid).

\section{Residual Errors}

Even after these corrections some residual errors will remain. These are controlled for by including the motion parameters (from realignment) into the design matrix, to soak up the 
variance caused by movement effects (Lund et al., 2005). However, adding an increasing number of nuisance regressors reduces the degrees of freedom and impacts the true signal, especially for shorter timeseries, as discussed in detail elsewhere (Bright and Murphy, 2015; Satterthwaite et al., 2013). Further, in SPM, a tool called ArtRepair (http:// cibsr.stanford.edu/tools/human-brain-project/artrepair-software/artrepairinstructions.html) can help to correct residual movement to reduce the need to include motion in the design matrix. ArtRepair can be used to interpolate outlier volumes, de-weight outliers only at the GLM level, or do both. Other tools are also available for such corrections (Ardekani et al., 2001; Oakes et al., 2005). However, as mentioned, including the motion parameters within the GLM can be problematic if the motion is correlated with the paradigm itself. Final analyses should be tested to see if motion is contributing to activation maps associated with variables of interest. Motion parameters can be examined between experimental factors (such as group, and condition) using statistical comparison tests such as 3dANOVA

\section{Other miscellaneous considerations}

Detrending (Byars et al., 2002; De Bie, 2010) can be performed at any stage and normalizes the data with respect to the mean of the time series. In pediatric populations the head size varies, especially with respect to the scanner and coil apparatus. This might cause any inhomogeneity in the magnetic, or other localized factors, might impact different parts of the brain in different participants, and also mediate the impact of movement

Voxel size is an important factor that might influence motion related processing. The acquisition voxel size is often used to influence the calculation of the threshold of unacceptable levels of volume-to-volume movement; with smaller voxel sizes it is possible that smaller degrees of motion could have a more noticeable impact and may thus merit a lower threshold (Johnstone et al., 2006). It is worth noting, however, that a number of groups have favored the use of mm over voxel-size in the measurement of volume-to-volume movement (e.g., Sattherthwaite, 2013; Power et al, 2015). Our voxel size is $3.4 \mathrm{~mm}$. We resampled this to $2 \mathrm{~mm}$ to match the standard templates. Although this slowed our processing considerably, the effect on motion correction and registration was superior because of the more fine-grained access to data. Smoothing is another important factor for motion correction as increased smoothing might impact ability to detect of motion, while also reduce the impact of small amount of motion on data. This should be decided based on the experimental questions, regions of interest, and population.

Developmental differences can also contribute to noise in the data in terms of increasing variability in brain activation that may decrease the ability to detect "true" between-group differences in activation maps. Thus, when working with adolescent and preadolescent populations it is important to assess the pubertal stage of participants (Blakemore et al., 2010; Luna et al., 2010). Depending on the age range, we should take the effect of age into consideration while looking at the neural activity. However, pubertal stage can be a more accurate marker of their developmental stage. When gathering pubertal data the most accurate measures are acquired through physiological markers (i.e., physical examination of the breasts and/or genitals; blood or saliva samples). However this might be inconvenient and off-putting for the participant, so many laboratories including our own use the Tanner 
Maturation Stage questionnaire (Marshall and Tanner, 1969, 1970) instead (Luna et al., 2010). This questionnaire is a self-report measure of secondary sexual characteristics associated with puberty.

In conclusion, fMRI data acquisition and analysis can be particularly susceptible to noise in pediatric and clinical pediatric groups. Much of this noise can be attributed to head motion. It is important to consider this at every stage of interaction with the pediatric participant, from recruitment to the consent process, to pre-scan training and during the fMRI scan itself. Once the researcher has acquired the best possible data by making all these careful considerations, there are a number of preprocessing steps that can be employed to further minimize the effect of noise. The most important step after data collection is to mindfully examine the results in detail and at every stage. Data with extreme motion effects should be eliminated. Data with moderate effects should be corrected, while examining output of every stage and eliminating data and subjects when necessary. Finally movement parameters should be included as nuisance variables in the design matrix as well as examined for contributing to significant group by condition results.

\section{Acknowledgments}

The authors would like to thank Tyler Lesh, Ph.D. for his input on the manuscript. We would also like to thank Steve Riley and Tadeus Arthur Hartanto for his assistance with data analysis. This work was supported by R01 MH091068 awarded to Julie B. Schweitzer and the UC Davis MIND Institute Intellectual and Developmental Disabilities Research Center (U54 HD079125).

\section{References}

Adjamian P, Barnes GR, Hillebrand A, Holliday IE, Singh KD, Furlong PL, Harrington E, Barclay $\mathrm{CW}$, Route PJ. Co-registration of magnetoencephalography with magnetic resonance imaging using bite-bar-based fiducials and surface-matching. Clin Neurophysiol. 2004; 115:691-698. [PubMed: 15036065]

Ardekani BA, Bachman AH, Helpern JA. A quantitative comparison of motion detection algorithms in fMRI. Magn Reson Imaging. 2001; 19:959-963. [PubMed: 11595367]

Beall EB, Lowe MJ. SimPACE: generating simulated motion corrupted BOLD data with syntheticnavigated acquisition for the development and evaluation of SLOMOCO: a new, highly effective slicewise motion correction. Neuroimage. 2014; 101:21-34. [PubMed: 24969568]

Bhattacharyya PK, Lowe MJ. Cardiac-induced physiologic noise in tissue is a direct observation of cardiac-induced fluctuations. Magn Reson Imaging. 2004; 22:9-13. [PubMed: 14972388]

Birn RM, Bandettini PA, Cox RW, Shaker R. Event-related fMRI of tasks involving brief motion. Hum Brain Mapp. 1999; 7:106-114. [PubMed: 9950068]

Birn RM, Cox RW, Bandettini PA. Experimental designs and processing strategies for fMRI studies involving overt verbal responses. Neuroimage. 2004; 23:1046-1058. [PubMed: 15528105]

Birn RM, Diamond JB, Smith MA, Bandettini PA. Separating respiratory-variation-related fluctuations from neuronal-activity-related fluctuations in fMRI. Neuroimage. 2006; 31:1536-1548. [PubMed: 16632379]

Blakemore SJ, Burnett S, Dahl RE. The role of puberty in the developing adolescent brain. Hum Brain Mapp. 2010; 31:926-933. [PubMed: 20496383]

Brett M, Johnsrude IS, Owen AM. The problem of functional localization in the human brain. Nat Rev Neurosci. 2002; 3:243-249. [PubMed: 11994756]

Bright MG, Murphy K. Reliable quantification of BOLD fMRI cerebrovascular reactivity despite poor breath-hold performance. Neuroimage. 2013; 83:559-568. [PubMed: 23845426] 
Bright MG, Murphy K. Is fMRI "noise" really noise? Resting state nuisance regressors remove variance with network structure. Neuroimage. 2015; 114:158-169. [PubMed: 25862264]

Bullmore ET, Brammer MJ, Rabe-Hesketh S, Curtis VA, Morris RG, Williams SC, Sharma T, McGuire PK. Methods for diagnosis and treatment of stimulus-correlated motion in generic brain activation studies using fMRI. Hum Brain Mapp. 1999; 7:38-48. [PubMed: 9882089]

Burgund ED, Kang HC, Kelly JE, Buckner RL, Snyder AZ, Petersen SE, Schlaggar BL. The feasibility of a common stereotactic space for children and adults in fMRI studies of development. Neuroimage. 2002; 17:184-200. [PubMed: 12482076]

Byars AW, Holland SK, Strawsburg RH, Bommer W, Dunn RS, Schmithorst VJ, Plante E. Practical aspects of conducting large-scale functional magnetic resonance imaging studies in children. $\mathrm{J}$ Child Neurol. 2002; 17:885-890. [PubMed: 12593460]

Centeno M, Tierney TM, Perani S, Shamshiri EA, StPier K, Wilkinson C, Konn D, Banks T, Vulliemoz S, Lemieux L, Pressler RM, Clark CA, Cross JH, Carmichael DW. Optimising EEGfMRI for Localisation of Focal Epilepsy in Children. PLoS One. 2016; 11:e0149048. [PubMed: 26872220]

Churchill NW, Oder A, Abdi H, Tam F, Lee W, Thomas C, Ween JE, Graham SJ, Strother SC. Optimizing preprocessing and analysis pipelines for single-subject fMRI. I. Standard temporal motion and physiological noise correction methods. Hum Brain Mapp. 2012; 33:609-627. [PubMed: 21455942]

Couvy-Duchesne B, Ebejer JL, Gillespie NA, Duffy DL, Hickie IB, Thompson PM, Martin NG, de Zubicaray GI, McMahon KL, Medland SE, Wright MJ. Head Motion and Inattention/ Hyperactivity Share Common Genetic Influences: Implications for fMRI Studies of ADHD. PLoS One. 2016:11.

Cox RW. AFNI: software for analysis and visualization of functional magnetic resonance neuroimages. Comput Biomed Res. 1996; 29:162-173. [PubMed: 8812068]

Cox RW. AFNI: what a long strange trip it's been. Neuroimage. 2012; 62:743-747. [PubMed: 21889996]

CurrentStudios, L. Don't Stumble Tumble. 2015.

Davids M, Zollner FG, Ruttorf M, Nees F, Flor H, Schumann G, Schad LR. Fully-automated quality assurance in multi-center studies using MRI phantom measurements. Magn Reson Imaging. 2014; 32:771-780. [PubMed: 24602825]

Davidson MC, Thomas KM, Casey BJ. Imaging the developing brain with fMRI. Ment Retard Dev Disabil Res Rev. 2003; 9:161-167. [PubMed: 12953295]

de Amorim e Silva CJ, Mackenzie A, Hallowell LM, Stewart SE, Ditchfield MR. Practice MRI: reducing the need for sedation and general anaesthesia in children undergoing MRI. Australas Radiol. 2006; 50:319-323. [PubMed: 16884416]

De Bie HMA, Boersma M, Wattjes MP, Adriaanse S, Vermeulen RJ, Oostrom KJ, ... Delemarre-Van de Waal HA. Preparing children with a mock scanner training protocol results in high quality structural and functional MRI scans. European Journal of Pediatrics. 2010; 169:1079-1085. [PubMed: 20225122]

Epstein JN, Casey BJ, Tonev ST, Davidson M, Reiss AL, Garrett A, Hinshaw SP, Greenhill LL, Vitolo A, Kotler LA, Jarrett MA, Spicer J. Assessment and prevention of head motion during imaging of patients with attention deficit hyperactivity disorder. Psychiatry Res. 2007; 155:75-82. [PubMed: 17395436]

Evans AC, Janke AL, Collins DL, Baillet S. Brain templates and atlases. Neuroimage. 2012; 62:911922. [PubMed: 22248580]

Fonov V, Evans AC, Botteron K, Almli CR, McKinstry RC, Collins DL. Unbiased average ageappropriate atlases for pediatric studies. Neuroimage. 2011; 54:313-327. [PubMed: 20656036]

Fox MD, Zhang D, Snyder AZ, Raichle ME. The global signal and observed anticorrelated resting state brain networks. J Neurophysiol. 2009; 101:3270-3283. [PubMed: 19339462]

Friston KJ, Holmes AP, Price CJ, Buchel C, Worsley KJ. Multisubject fMRI studies and conjunction analyses. Neuroimage. 1999; 10:385-396. [PubMed: 10493897]

Friston KJ, Williams S, Howard R, Frackowiak RS, Turner R. Movement-related effects in fMRI timeseries. Magn Reson Med. 1996; 35:346-355. [PubMed: 8699946] 
Galvan A. Adolescent development of the reward system. Front Hum Neurosci. 2010; 4:6. [PubMed: 20179786]

Galvan A, Van Leijenhorst L, McGlennen KM. Considerations for imaging the adolescent brain. Dev Cogn Neurosci. 2012; 2:293-302. [PubMed: 22669033]

Greve DN, Brown GG, Mueller BA, Glover G, Liu TT. A survey of the sources of noise in fMRI. Psychometrika. 2013; 78:396-416. [PubMed: 25106392]

Grier RA, Warm JS, Dember WN, Matthews G, Galinsky TL, Parasuraman R. The vigilance decrement reflects limitations in effortful attention, not mindlessness. Hum Factors. 2003; 45:349359. [PubMed: 14702988]

Griffanti L, Salimi-Khorshidi G, Beckmann CF, Auerbach EJ, Douaud G, Sexton CE, Zsoldos E, Ebmeier KP, Filippini N, Mackay CE, Moeller S, Xu J, Yacoub E, Baselli G, Ugurbil K, Miller KL, Smith SM. ICA-based artefact removal and accelerated fMRI acquisition for improved resting state network imaging. Neuroimage. 2014; 95:232-247. [PubMed: 24657355]

Hartanto TA, Krafft CE, Iosif AM, Schweitzer JB. A trial-by-trial analysis reveals more intense physical activity is associated with better cognitive control performance in attention-deficit/ hyperactivity disorder. Child Neuropsychol. 2016; 22:618-626. [PubMed: 26059476]

Helton WS, Hollander TD, Warm JS, Matthews G, Dember WN, Wallaart M, Beauchamp G, Parasuraman R, Hancock PA. Signal regularity and the mindlessness model of vigilance. Br J Psychol. 2005; 96:249-261. [PubMed: 15969834]

Hong LE, Gu H, Yang Y, Ross TJ, Salmeron BJ, Buchholz B, Thaker GK, Stein EA. Association of nicotine addiction and nicotine's actions with separate cingulate cortex functional circuits. Arch Gen Psychiatry. 2009; 66:431-441. [PubMed: 19349313]

Huang CM, Lee SH, Hsiao IT, Kuan WC, Wai YY, Ko HJ, Wan YL, Hsu YY, Liu HL. Study-specific EPI template improves group analysis in functional MRI of young and older adults. J Neurosci Methods. 2010; 189:257-266. [PubMed: 20346979]

Johnstone T, Ores Walsh KS, Greischar LL, Alexander AL, Fox AS, Davidson RJ, Oakes TR. Motion correction and the use of motion covariates in multiple-subject fMRI analysis. Hum Brain Mapp. 2006; 27:779-788. [PubMed: 16456818]

Kang HC, Burgund ED, Lugar HM, Petersen SE, Schlaggar BL. Comparison of functional activation foci in children and adults using a common stereotactic space. Neuroimage. 2003; 19:16-28. [PubMed: 12781724]

Kotsoni E, Byrd D, Casey BJ. Special considerations for functional magnetic resonance imaging of pediatric populations. J Magn Reson Imaging. 2006; 23:877-886. [PubMed: 16649204]

Kundu P, Benson BE, Baldwin KL, Rosen D, Luh WM, Bandettini PA, Pine DS, Ernst M. Robust resting state fMRI processing for studies on typical brain development based on multi-echo EPI acquisition. Brain Imaging Behav. 2015; 9:56-73. [PubMed: 25592183]

Lee JS, Lee DS, Kim J, Kim YK, Kang E, Kang H, Kang KW, Lee JM, Kim JJ, Park HJ, Kwon JS, Kim SI, Yoo TW, Chang KH, Lee MC. Development of Korean standard brain templates. J Korean Med Sci. 2005; 20:483-488. [PubMed: 15953874]

Lucker JR, Geffner D, Koch W. Perception of loudness in children with ADD and without ADD. Child Psychiatry Hum Dev. 1996; 26:181-190. [PubMed: 8819880]

Luking KR, Pagliaccio D, Luby JL, Barch DM. Depression Risk Predicts Blunted Neural Responses to Gains and Enhanced Responses to Losses in Healthy Children. J Am Acad Child Adolesc Psychiatry. 2016; 55:328-337. [PubMed: 27015724]

Luna B, Velanova K, Geier CF. Methodological approaches in developmental neuroimaging studies. Hum Brain Mapp. 2010; 31:863-871. [PubMed: 20496377]

Lund TE, Norgaard MD, Rostrup E, Rowe JB, Paulson OB. Motion or activity: their role in intra- and inter-subject variation in fMRI. Neuroimage. 2005; 26:960-964. [PubMed: 15955506]

Maclaren J, Herbst M, Speck O, Zaitsev M. Prospective motion correction in brain imaging: a review. Magn Reson Med. 2013; 69:621-636. [PubMed: 22570274]

Mandal PK, Mahajan R, Dinov ID. Structural brain atlases: design, rationale, and applications in normal and pathological cohorts. J Alzheimers Dis. 2012; 31(Suppl 3):S169-188. [PubMed: 22647262] 
Marshall WA, Tanner JM. Variations in pattern of pubertal changes in girls. Arch Dis Child. 1969; 44:291-303. [PubMed: 5785179]

Marshall WA, Tanner JM. Variations in the pattern of pubertal changes in boys. Arch Dis Child. 1970; 45:13-23. [PubMed: 5440182]

Mazaika, P., Hoeft, F., Glover, GH., Reiss, AL. Methods and Software for fMRI Analysis for Clinical Subjects. Organization for Human Brain Mapping; San Francisco, CA, USA: 2009.

Mazaika, P., Whitfield-Gabrieli, S., Reiss, AL. Artifact Repair for fMRI Data from High Motion Clinical Subjects. Organization for Human Brain Mapping; Chicago, IL, USA: 2007.

Maziero D, Velasco TR, Hunt N, Payne E, Lemieux L, Salmon CE, Carmichael DW. Towards motion insensitive EEG-fMRI: Correcting motion-induced voltages and gradient artefact instability in EEG using an fMRI prospective motion correction (PMC) system. Neuroimage. 2016; 138:13-27. [PubMed: 27157789]

Muresan L, Renken R, Roerdink JB, Duifhuis H. Automated correction of spin-history related motion artefacts in fMRI: simulated and phantom data. IEEE Trans Biomed Eng. 2005; 52:1450-1460. [PubMed: 16119241]

Murphy K, Birn RM, Bandettini PA. Resting-state fMRI confounds and cleanup. Neuroimage. 2013; 80:349-359. [PubMed: 23571418]

Murphy K, Birn RM, Bandettini PA. Resting-state FMRI confounds and cleanup. Neuroimage. 2013; 80:349-359. [PubMed: 23571418]

Murphy K, Garavan H. Deriving the optimal number of events for an event-related fMRI study based on the spatial extent of activation. Neuroimage. 2005; 27:771-777. [PubMed: 15961321]

Nordahl CW, Mello M, Shen AM, Shen MD, Vismara LA, Li D, Harrington K, Tanase C, GoodlinJones B, Rogers S, Abbeduto L, Amaral DG. Methods for acquiring MRI data in children with autism spectrum disorder and intellectual impairment without the use of sedation. J Neurodev Disord. 2016; 8:20. [PubMed: 27158271]

Oakes TR, Johnstone T, Ores Walsh KS, Greischar LL, Alexander AL, Fox AS, Davidson RJ. Comparison of fMRI motion correction software tools. Neuroimage. 2005; 28:529-543. [PubMed: 16099178]

Ollinger JMOT, Alexander AL, Haeberli F, Dalton KM, Davidson RJ. The secret life of motion covariates. Neuroimage. 2009:47. [PubMed: 19457371]

Padilla N, Falcon C, Sanz-Cortes M, Figueras F, Bargallo N, Crispi F, Eixarch E, Arranz A, Botet F, Gratacos E. Differential effects of intrauterine growth restriction on brain structure and development in preterm infants: a magnetic resonance imaging study. Brain Res. 2011; 1382:98108. [PubMed: 21255560]

Park DC, Reuter-Lorenz P. The adaptive brain: aging and neurocognitive scaffolding. Annu Rev Psychol. 2009; 60:173-196. [PubMed: 19035823]

Pernet CR. Misconceptions in the use of the General Linear Model applied to functional MRI: a tutorial for junior neuro-imagers. Front Neurosci. 2014:8. [PubMed: 24550770]

Poldrack RA, Pare-Blagoev EJ, Grant PE. Pediatric functional magnetic resonance imaging: progress and challenges. Top Magn Reson Imaging. 2002; 13:61-70. [PubMed: 11847501]

Power JD, Barnes KA, Snyder AZ, Schlaggar BL, Petersen SE. Steps toward optimizing motion artifact removal in functional connectivity MRI; a reply to Carp. Neuroimage. 2013; 76:439-441. [PubMed: 22440651]

Power JD, Schlaggar BL, Petersen SE. Recent progress and outstanding issues in motion correction in resting state fMRI. Neuroimage. 2015; 105:536-551. [PubMed: 25462692]

Raschle N, Zuk J, Ortiz-Mantilla S, Sliva DD, Franceschi A, Grant PE, Benasich AA, Gaab N. Pediatric neuroimaging in early childhood and infancy: challenges and practical guidelines. Ann N Y Acad Sci. 2012; 1252:43-50. [PubMed: 22524338]

Raschle N, Zuk J, Ortiz-Mantilla S, Sliva DD, Franceschi A, Grant PE, Benasich AA, Gaab N. Pediatric neuroimaging in early childhood and infancy: challenges and practical guidelines. Annals of the New York Academy of Sciences. 2012; 1252:43-50. [PubMed: 22524338]

Rohlfing TSE, Pfefferbaum A. Subject-Matched Templates for Spatial Normalization. Medical Image Computing and Computer-Assisted Intervention: MICCAI. 2012:1-10. 
Salimi-Khorshidi G, Douaud G, Beckmann CF, Glasser MF, Griffanti L, Smith SM. Automatic denoising of functional MRI data: combining independent component analysis and hierarchical fusion of classifiers. Neuroimage. 2014; 90:449-468. [PubMed: 24389422]

Sanchez CE, Richards JE, Almli CR. Age-specific MRI templates for pediatric neuroimaging. Dev Neuropsychol. 2012; 37:379-399. [PubMed: 22799759]

Sarkka S, Solin A, Nummenmaa A, Vehtari A, Auranen T, Vanni S, Lin FH. Dynamic retrospective filtering of physiological noise in BOLD fMRI: DRIFTER. Neuroimage. 2012; 60:1517-1527. [PubMed: 22281675]

Satterthwaite TD, Elliott MA, Gerraty RT, Ruparel K, Loughead J, Calkins ME, Eickhoff SB, Hakonarson H, Gur RC, Gur RE, Wolf DH. An improved framework for confound regression and filtering for control of motion artifact in the preprocessing of resting-state functional connectivity data. Neuroimage. 2013; 64:240-256. [PubMed: 22926292]

Schlund MW, Cataldo MF, Siegle GJ, Ladouceur CD, Silk JS, Forbes EE, McFarland A, Iyengar S, Dahl RE, Ryan ND. Pediatric functional magnetic resonance neuroimaging: tactics for encouraging task compliance. Behav Brain Funct. 2011; 7:10. [PubMed: 21548928]

Schweitzer JB, Sulzer-Azaroff B. Self-control: teaching tolerance for delay in impulsive children. J Exp Anal Behav. 1988; 50:173-186. [PubMed: 3193054]

Schweitzer JB, Sulzer-Azaroff B. Self-control in boys with attention deficit hyperactivity disorder: effects of added stimulation and time. J Child Psychol Psychiatry. 1995; 36:671-686. [PubMed: 7650090]

Siegel JS, Power JD, Dubis JW, Vogel AC, Church JA, Schlaggar BL, Petersen SE. Statistical improvements in functional magnetic resonance imaging analyses produced by censoring highmotion data points. Hum Brain Mapp. 2014; 35:1981-1996. [PubMed: 23861343]

Slifer KJ, Cataldo MF, Cataldo MD, Llorente AM, Gerson AC. Behavior analysis of motion control for pediatric neuroimaging. J Appl Behav Anal. 1993; 26:469-470. [PubMed: 8307831]

Slifer KJ, Koontz KL, Cataldo MF. Operant-contingency-based preparation of children for functional magnetic resonance imaging. J Appl Behav Anal. 2002; 35:191-194. [PubMed: 12102139]

Slifer KJ, Penn-Jones K, Cataldo MF, Conner RT, Zerhouni EA. Music enhances patients' comfort during MR imaging. AJR Am J Roentgenol. 1991; 156:403.

Szaflarski JP, Holland SK, Schmithorst VJ, Byars AW. fMRI study of language lateralization in children and adults. Hum Brain Mapp. 2006; 27:202-212. [PubMed: 16035047]

Temple E, Poldrack RA, Salidis J, Deutsch GK, Tallal P, Merzenich MM, Gabrieli JD. Disrupted neural responses to phonological and orthographic processing in dyslexic children: an fMRI study. Neuroreport. 2001; 12:299-307. [PubMed: 11209939]

Thomason ME. Children in Non-Clinical Functional Magnetic Resonance Imaging (fMRI) Studies Give the Scan Experience a "Thumbs Up”. American Journal of Bioethics. 2009; 9:25-27.

Tierney TM, Weiss-Croft LJ, Centeno M, Shamshiri EA, Perani S, Baldeweg T, Clark CA, Carmichael DW. FIACH: A biophysical model for automatic retrospective noise control in fMRI. Neuroimage. 2016; 124:1009-1020. [PubMed: 26416652]

Todd N, Josephs O, Callaghan MF, Lutti A, Weiskopf N. Prospective motion correction of 3D echoplanar imaging data for functional MRI using optical tracking. Neuroimage. 2015; 113:1-12. [PubMed: 25783205]

Turner BO, Miller MB. Number of events and reliability in fMRI. Cogn Affect Behav Neurosci. 2013; 13:615-626. [PubMed: 23754543]

Vaidya CJ, Austin G, Kirkorian G, Ridlehuber HW, Desmond JE, Glover GH, Gabrieli JD. Selective effects of methylphenidate in attention deficit hyperactivity disorder: a functional magnetic resonance study. Proc Natl Acad Sci USA. 1998; 95:14494-14499. [PubMed: 9826728]

Van Horn JD, Pelphrey KA. Neuroimaging of the developing brain. Brain Imaging Behav. 2015; 9:1-4. [PubMed: 25724689]

Weinberger DR, Radulescu E. Finding the Elusive Psychiatric "Lesion" With 21st-Century Neuroanatomy: A Note of Caution. Am J Psychiatry. 2016; 173:27-33. [PubMed: 26315983]

Weissenbacher A, Kasess C, Gerstl F, Lanzenberger R, Moser E, Windischberger C. Correlations and anticorrelations in resting-state functional connectivity MRI: a quantitative comparison of preprocessing strategies. Neuroimage. 2009; 47:1408-1416. [PubMed: 19442749] 
Weissman DG, Schriber RA, Fassbender C, Atherton O, Krafft C, Robins RW, Hastings PD, Guyer AE. Earlier adolescent substance use onset predicts stronger connectivity between reward and cognitive control brain networks. Dev Cogn Neurosci. 2015; 16:121-129. [PubMed: 26215473]

Wilke M, Holland SK, Myseros JS, Schmithorst VJ, Ball WS. Functional magnetic resonance imaging in pediatrics. Neuropediatrics. 2003; 34:225-233. [PubMed: 14598227]

Wilke M, Schmithorst VJ, Holland SK. Assessment of spatial normalization of whole-brain magnetic resonance images in children. Hum Brain Mapp. 2002; 17:48-60. [PubMed: 12203688]

Wilke M, Schmithorst VJ, Holland SK. Normative pediatric brain data for spatial normalization and segmentation differs from standard adult data. Magn Reson Med. 2003; 50:749-757. [PubMed: 14523961]

Wylie GR, Genova H, DeLuca J, Chiaravalloti N, Sumowski JF. Functional magnetic resonance imaging movers and shakers: does subject-movement cause sampling bias? Hum Brain Mapp. 2014; 35:1-13. [PubMed: 22847906]

Yancey SE, Rotenberg DJ, Tam F, Chiew M, Ranieri S, Biswas L, Anderson KJ, Baker SN, Wright GA, Graham SJ. Spin-history artifact during functional MRI: potential for adaptive correction. Med Phys. 2011; 38:4634-4646. [PubMed: 21928636]

Zhang Y, Zhang J, Hsu J, Oishi K, Faria AV, Albert M, Miller MI, Mori S. Evaluation of groupspecific, whole-brain atlas generation using Volume-based Template Estimation (VTE): application to normal and Alzheimer's populations. Neuroimage. 2014; 84:406-419. [PubMed: 24051356] 

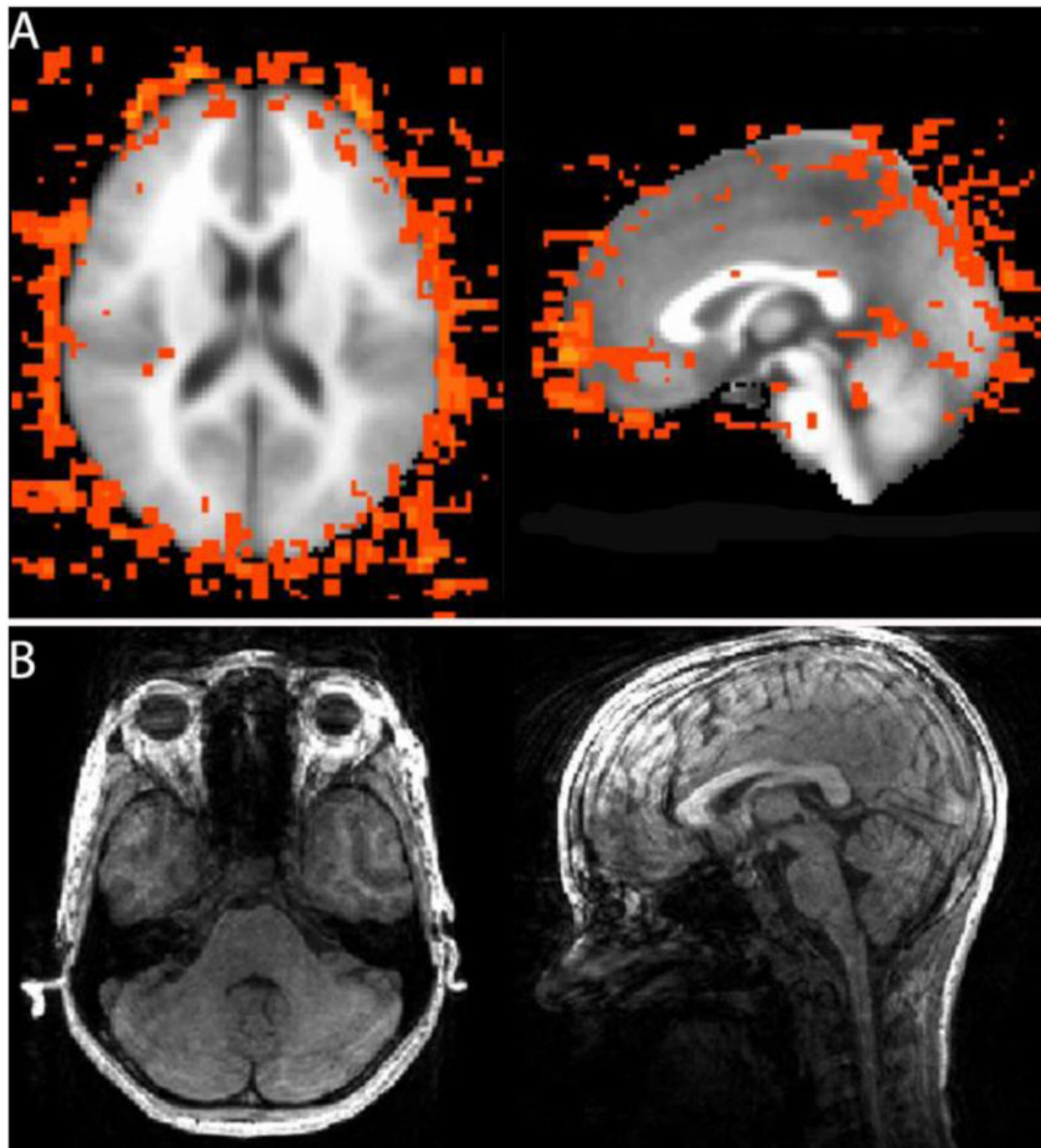

Figure 1.

An example from our laboratory of a subject displaying excessive motion (this subject had $19.2 \%$ of TRs censored due to movement that exceeded our acceptable threshold).

Examination of the activation map (A) revealed activity around the edges of the brain and in brain regions particularly susceptible to movement artifact. The subject's anatomy (B) was also contaminated by motion artifacts (ring-like pattern visible in the saggital view). 


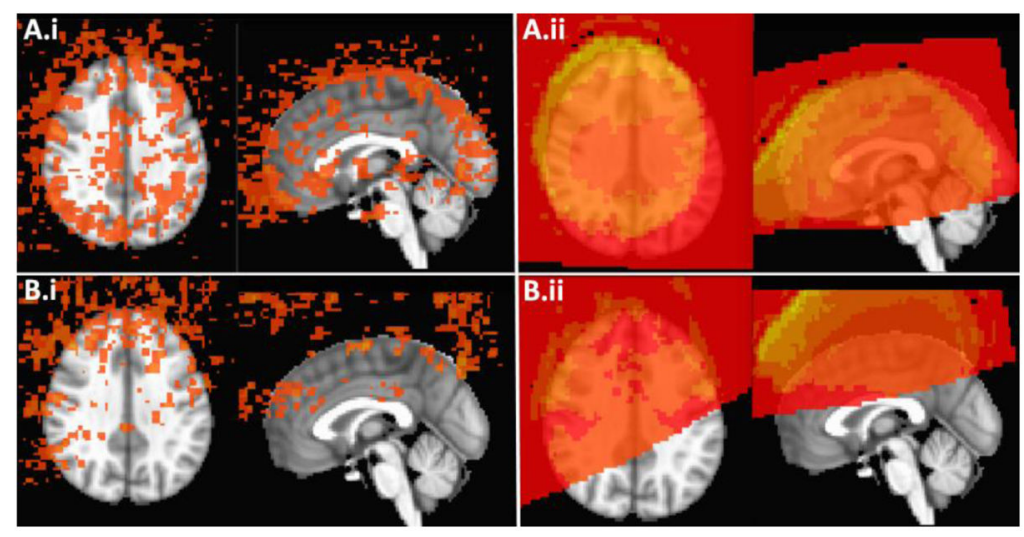

Figure 2.

Two examples of subjects with poor registration. Like the example in Figure 1, both subjects A (i) and B (i) displayed excessive amounts of activation outside compared with inside the brain boundary. However, closer inspection revealed (Aii and Bii) that this was likely due to poor registration of the EPI; the activation map does not align well with the anatomy, particularly in the case of subject B. 
Table 1

Data from our laboratory on number of scans completed in typically developing (TD) and ADHD groups.

\begin{tabular}{lrr}
\hline scans & TD & ADHD \\
\hline Total & 155 & 178 \\
Aborted & 1 & 0 \\
Incomplete - out of time & 5 & 9 \\
Restarted runs (reminder of instructions or excessive motion) & 13 & 23 \\
exiting the scanner to use restroom or move around & 11 & 21 \\
\hline
\end{tabular}




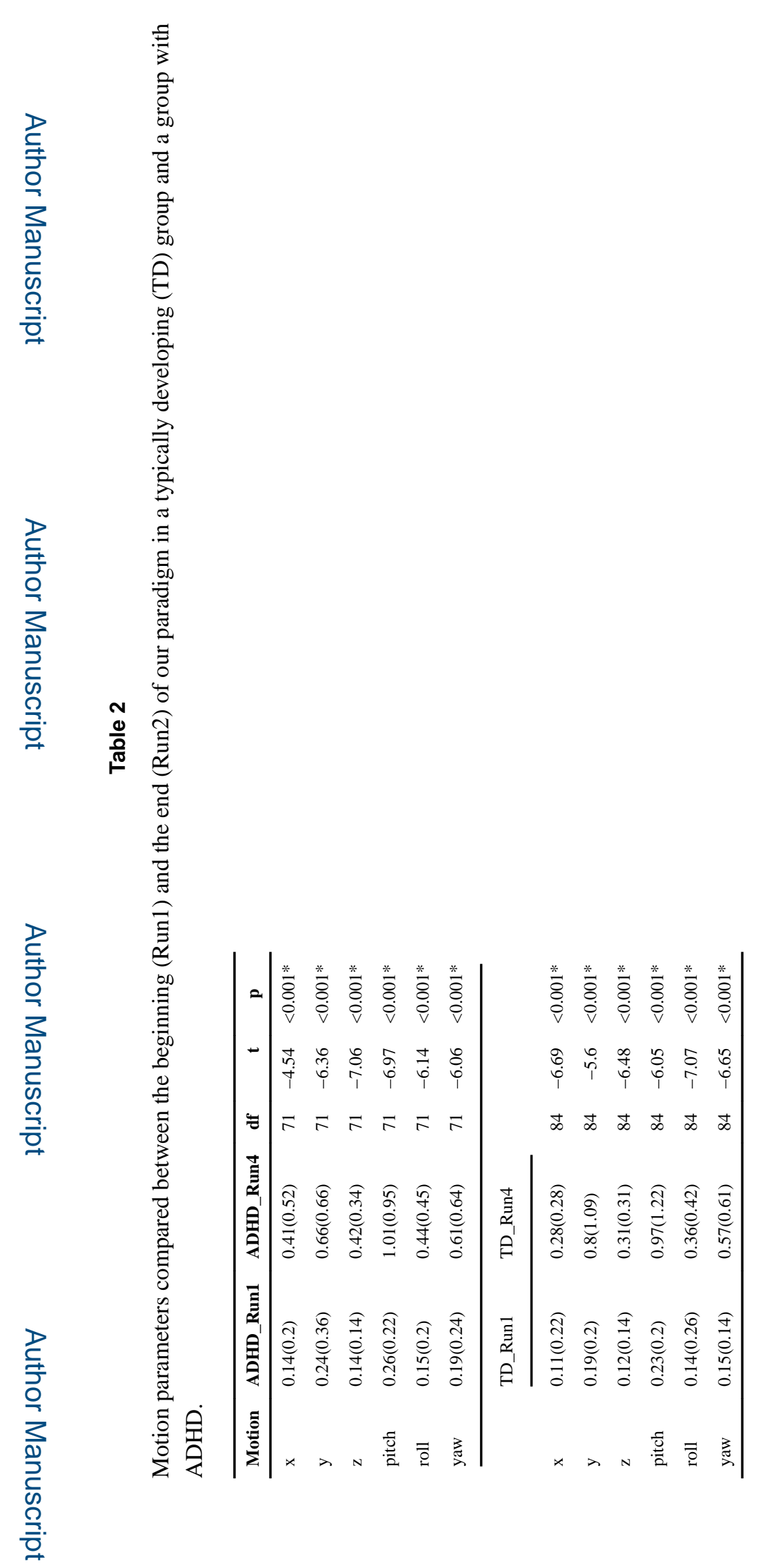

Neuroimage. Author manuscript; available in PMC 2018 April 01. 


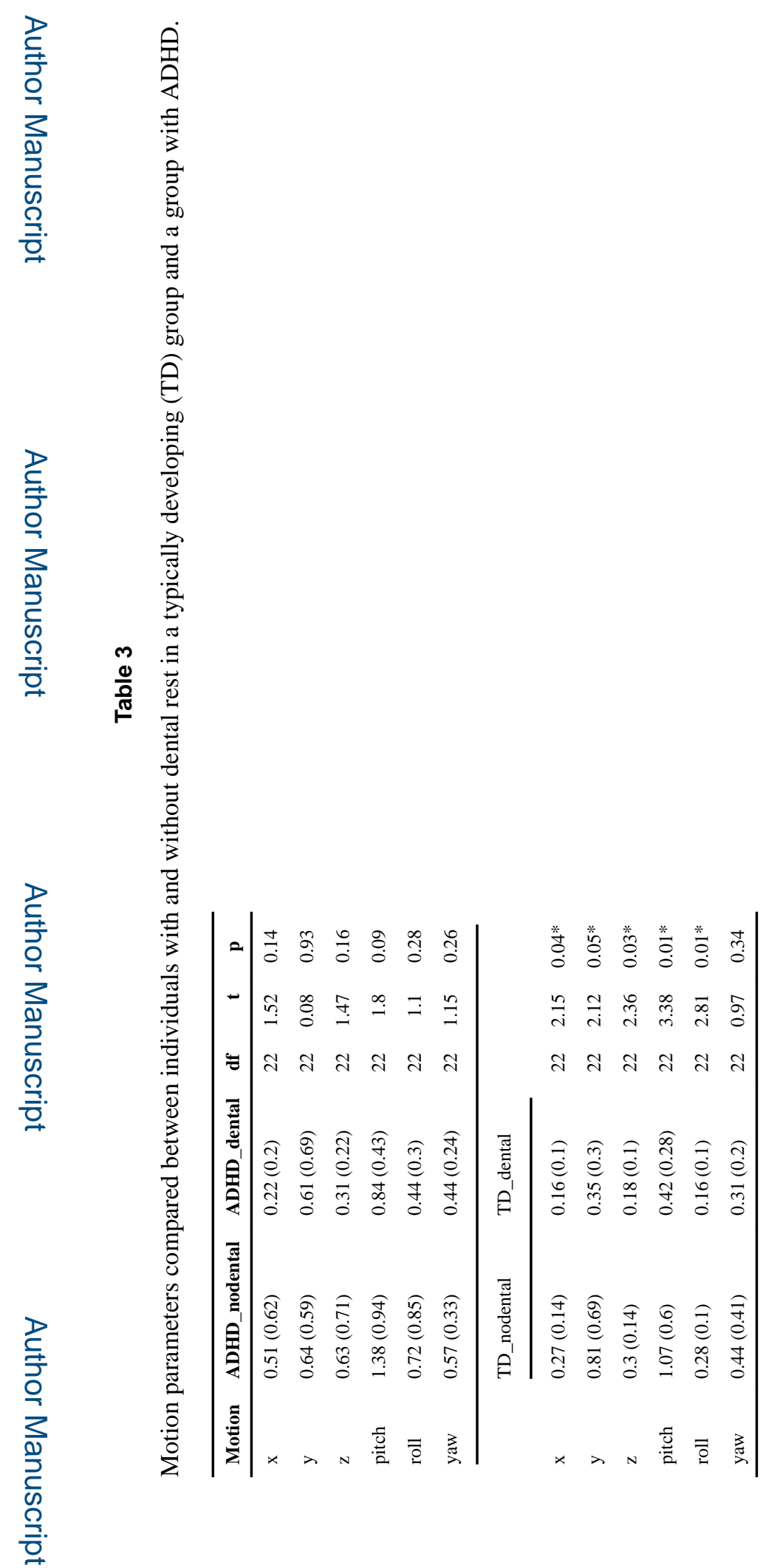

Neuroimage. Author manuscript; available in PMC 2018 April 01. 